\title{
SOLUTION OF TIME-DEPENDENT ADVECTION-DIFFUSION PROBLEMS WITH THE SPARSE-GRID COMBINATION TECHNIQUE AND A ROSENBROCK SOLVER ${ }^{1}$
}

\author{
BORIS LASTDRAGER, BARRY KOREN, AND JAN VERWER \\ CWI, Center for Mathematics and Computer Science \\ P.O. Box 94079, 1090 GB Amsterdam, The Netherlands \\ E-mail: boris.lastdrager@cwi.nl, barry.koren@cwi.nl, jan.verwer@cwi.nl
}

\begin{abstract}
In the current paper the efficiency of the sparse-grid combination technique applied to time-dependent advection-diffusion problems is investigated. For the time-integration we employ a third-order Rosenbrock scheme implemented with adaptive step-size control and approximate matrix factorization. Two model problems are considered, a scalar 2D linear, constant-coefficient problem and a system of 2D nonlinear Burgers' equations. In short, the combination technique proved more efficient than a single grid approach for the simpler linear problem. For the Burgers' equations this gain in efficiency was only observed if one of the two solution components was set to zero, which makes the problem more grid-aligned.
\end{abstract}

2000 Mathematics Subject Classification: 65G99, 65M20, 65M55, 65L06, 76R99.

Keywords: advection-diffusion problems, sparse grids, combination techniques, Rosenbrock methods, error analysis.

\section{Introduction}

In modern $\mathrm{CFD}$ codes accurate resolution of thin solution layers is still very time consuming. Especially for high Reynolds numbers many grid points are needed to resolve very thin layers. The common remedy is to use adapted grids that have small cells near the layers and large cells elsewhere. In this paper we investigate another approach to resolve the thin layers, namely the sparse grid combination technique (CT) as introduced by Griebel, Schneider and Zenger [4].

The $\mathrm{CT}$ is attractive because, asymptotically, it can yield a smaller spatial error for a given complexity than a single grid approach $(\mathrm{SG})$ can $[1,14]$. Consider a problem of spatial dimension $d$ that is solved on a single grid with spatial discretization of order $p$, i.e., on a single grid with mesh-width $h$ the spatial error is $O\left(h^{p}\right)$. On a single grid this problem would have a complexity $\sim h^{-d}$. With the CT a spatial error of order $O\left(h^{p}(\log h)^{d-1}\right)$ can be obtained with a complexity $\sim h^{-1}(\log h)^{d-1}$, i.e., an asymptotically first-order complexity is obtained with only a slightly larger error than for the SG. Furthermore, the CT can be easily and efficiently implemented on a parallel computer, see [3].

\footnotetext{
${ }^{1}$ This work was performed under a research contract with The Netherlands Organization for Scientific Research (NWO) and was carried out under CWI-projects MAS1.1 "Numerical Algorithms for Air Quality Modeling" and MAS2.1 "Computational Fluid Dynamics".
} 
In $[10]$ we investigated the efficiency of the CT when applied to a pure advection equation and concluded that for a non-grid-aligned solution the CT does not perform very well. In [11] this was also found for some elliptic PDEs. Note that in [5] the CT is also applied to a pure advection equation, but there the efficiency of the CT is not considered.

In practice, advection-diffusion problems are usually solved on boundary-fitted grids. The corresponding solutions are usually grid-aligned. Also in this paper we study model advection-diffusion problems having this type of solution.

An essential ingredient for a CT solver for time-dependent problems is an efficient time accurate integrator. We use a three-stage, third-order Rosenbrock method, implemented with built-in step-size control and approximate matrix factorization. Without step-size control the method can be implemented as a two-stage scheme. It uses approximate matrix factorization to greatly speed up the solution process. Hence we call it factorized ROS3. Independently from the current paper, in [7] the same factorized ROS3 has been used without the CT.

As model problems we consider a scalar two-dimensional, constant-coefficient advectiondiffusion equation and a system of two-dimensional Burgers' equations. To evaluate the efficiency of the CT we compare it with a straightforward SG approach.

\section{The model problems}

\subsection{Model problem 1: The advection-diffusion equation}

We consider the constant-coefficient advection-diffusion equation

$$
u_{t}+u_{x}-\varepsilon\left(u_{x x}+u_{y y}\right)=0
$$

on the spatial domain $[-1,1] \times[-1,1]$ and take $u(x, y, 0)=0$ as initial solution. As boundary conditions we impose

$$
u(-1, y, t)=\left\{\begin{array}{ll}
0, & y<0 \\
\frac{1}{2}, & y=0 \\
1, & y>0
\end{array}, \quad u_{y}(x, \pm 1, t)=0, \quad u(1, y, t)=0 .\right.
$$

For $\varepsilon=10^{-2}$ the solution at $t=1$ is shown in Fig. 1. It possesses a horizontal and a vertical grid-aligned solution layers. The thickness of both layers is proportional to $\sqrt{\varepsilon}$ as $\varepsilon \rightarrow 0$. For the steady state solution we have derived an exact expression in terms of a Fourier sum,

$$
\begin{aligned}
& u(x, y)=\frac{3 / 2}{\left(1-e^{1 / \varepsilon}\right)} e^{x / \varepsilon}\left(1-e^{(1-x) / \varepsilon}\right)+\sum_{n=1}^{\infty} B_{n}(x) \cos (n \pi y), \\
& B_{n}(x)=\frac{2 \sin \left(\frac{n \pi}{2}\right) / n \pi}{e^{2 \sqrt{1 /\left(4 \varepsilon^{2}\right)+n^{2} \pi^{2}}}-1} e^{x /(2 \varepsilon)}\left(e^{x \sqrt{1 /\left(4 \varepsilon^{2}\right)+n^{2} \pi^{2}}}-e^{(2-x) \sqrt{\frac{1}{4 \varepsilon^{2}}+n^{2} \pi^{2}}}\right),
\end{aligned}
$$

and have used this expression to confirm that our numerical method converges to the correct solution in the limit $t \rightarrow \infty$.

\subsection{Model problem 2: Burgers' equations}

The two-dimensional system of Burgers' equations

$$
\begin{aligned}
& u_{t}=-u u_{x}-v u_{y}+\varepsilon\left(u_{x x}+u_{y y}\right), \\
& v_{t}=-u v_{x}-v v_{y}+\varepsilon\left(v_{x x}+v_{y y}\right)
\end{aligned}
$$




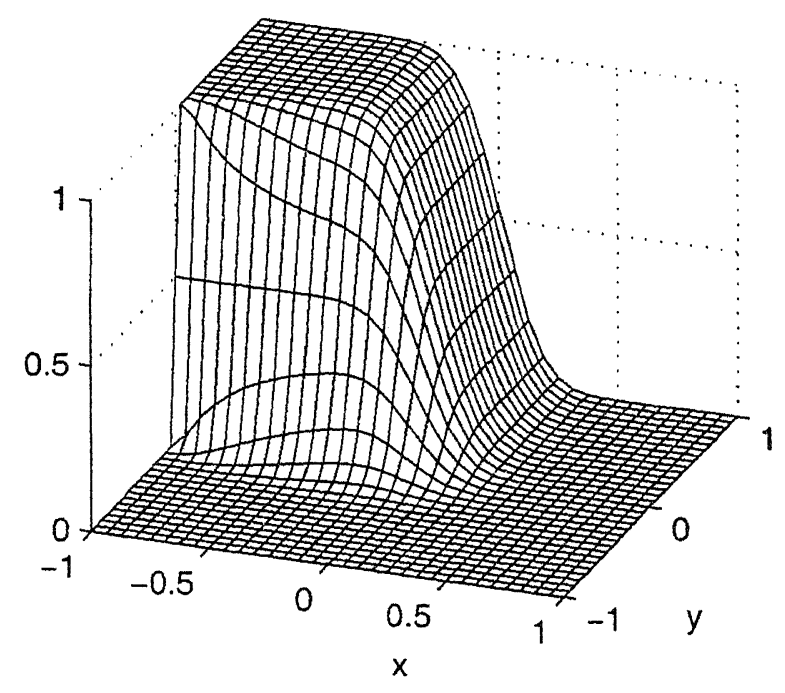

Figure 1. Solution of model problem 1 at $t=1$ for $\epsilon=0.01$.

is considered on the spatial domain $[-1,1] \times[-1,1]$. The boundary conditions for $t>0$ are

$$
u(-1, y, t)=\left\{\begin{array}{l}
1-4(y-1 / 2)^{2}, y \geqslant 0 \\
1-4(y+1 / 2)^{2}, y<0
\end{array}, \quad u(x, \pm 1, t)=0, \quad u_{x}(1, y, t)=0,\right.
$$

and

$$
v(-1, y, t)=-0.35 \sin \left(\frac{1}{2} \pi y\right), \quad v_{y}(x, \pm 1, t)=0, \quad v_{x}(1, y, t)=0
$$

As initial solutions we take

$$
\begin{aligned}
& u(x, y, 0)=\left\{\begin{array}{l}
1-4(y-1 / 2)^{2}, y \geqslant 0 \\
1-4(y+1 / 2)^{2}, y<0
\end{array},\right. \\
& v(x, y, 0)=-0.35 \sin \left(\frac{1}{2} \pi y\right) .
\end{aligned}
$$

In Figs. 2 and 3 the $u$ and $v$ components of the solution at $t=3$ are shown for $\varepsilon=10^{-2}$. The $v$ component shows sharpening from the sinusoidal inlet condition at $x=-1$ to a much steeper slope at the outflow boundary at $x=1$. This is a grid-aligned phenomenon, since near the outflow boundary the solution varies much stronger in the $y$ direction than in the $x$ one. The $u$ component shows a mixing of two jets. This phenomenon is not especially grid-aligned, since variations in the $x$ and the $y$ directions are comparable.

\section{The sparse grid combination technique}

In the CT several solutions on different grids are combined to obtain a solution which has the accuracy corresponding with a much finer grid. The two-dimensional CT is based on a family of grids as shown in Fig. 4. Grids in the family of grids are denoted by $\Omega^{l, m}$ where the superscripts label the level of refinement relative to the root grid $\Omega^{0,0}$. The mesh-widths in the $x$ and $y$ directions of $\Omega^{l, m}$ are $h_{x}=2^{-l} H$ and $h_{y}=2^{-m} H$, where $H$ is the mesh width of the uniform root grid $\Omega^{0,0}$. We denote the mesh width of the finest grid $\Omega^{N, N}$ by $h$. Note that $h_{x}$ and $h_{y}$ are dependent on the particular grid $\Omega^{l, m}$ in the family of grids, whereas $h$ is not. 


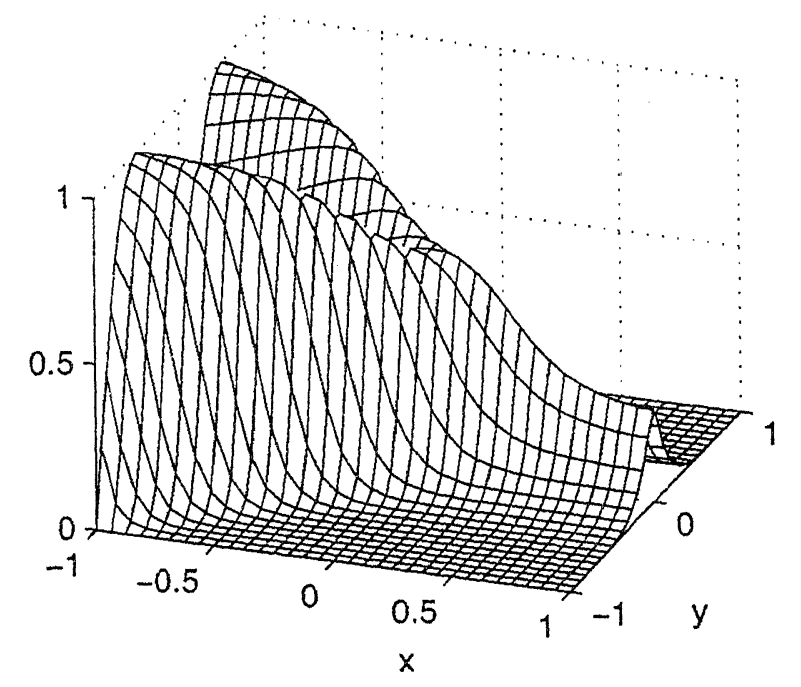

Figure 2. $u$-Component of the solution of model problem 2 at $t=3$ for $\epsilon=0.01$.

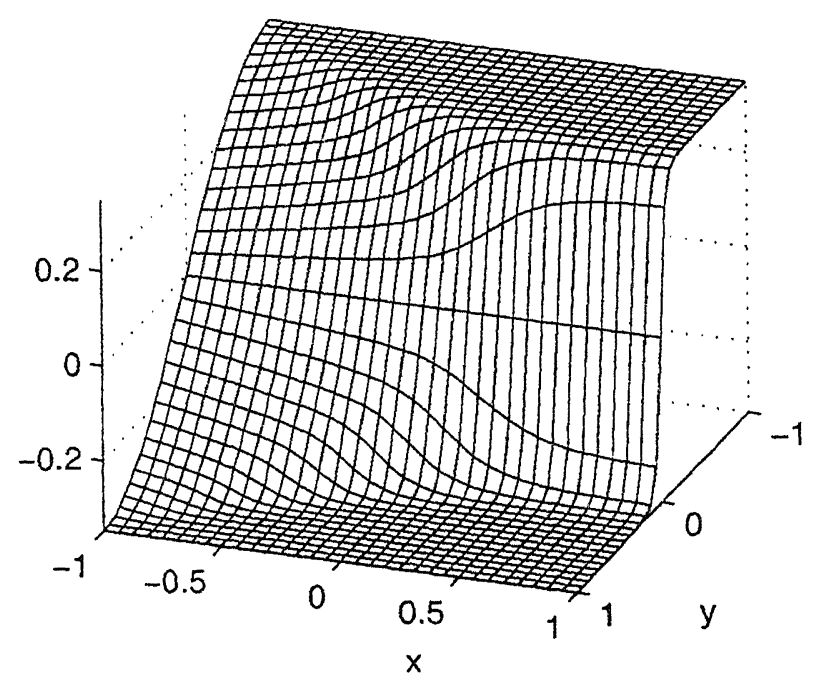

Figure 3. $v$-Component of the solution of model problem 2 at $t=3$ for $\epsilon=0.01$.

In the time-dependent combination technique the initial profile $u(x, y, 0)$ is restricted, by injection, to the grids $\Omega^{N, 0}, \Omega^{N-1,1}, \cdots, \Omega^{0, N}$ and to $\Omega^{N-1,0}, \Omega^{N-2,1}, \cdots, \Omega^{0, N-1}$, see Fig. 4 . Then, independent of each other, these rather coarse representations are all integrated in time by our ROS3 time integrator. Then, at a chosen point in time, the coarse approximations are prolongated with $q$-th order interpolation onto the finest grid $\Omega^{N, N}$, where the integrated solutions are combined to obtain a more accurate solution. The notation is summarized in Fig. 4.

Starting from the exact solution $u$, the combination technique, as introduced in [4], constructs a grid function $\widehat{u}^{N, N}$ on the finest grid $\Omega^{N, N}$ in the following manner:

$$
\widehat{u}^{N, N} \equiv \sum_{l+m=N} P^{N, N} R^{l, m} u-\sum_{l+m=N-1} P^{N, N} R^{l, m} u
$$

The corresponding so-called representation error $r^{N, N}$ is

$$
r^{N, N} \equiv \widehat{u}^{N, N}-R^{N, N} u \text {. }
$$




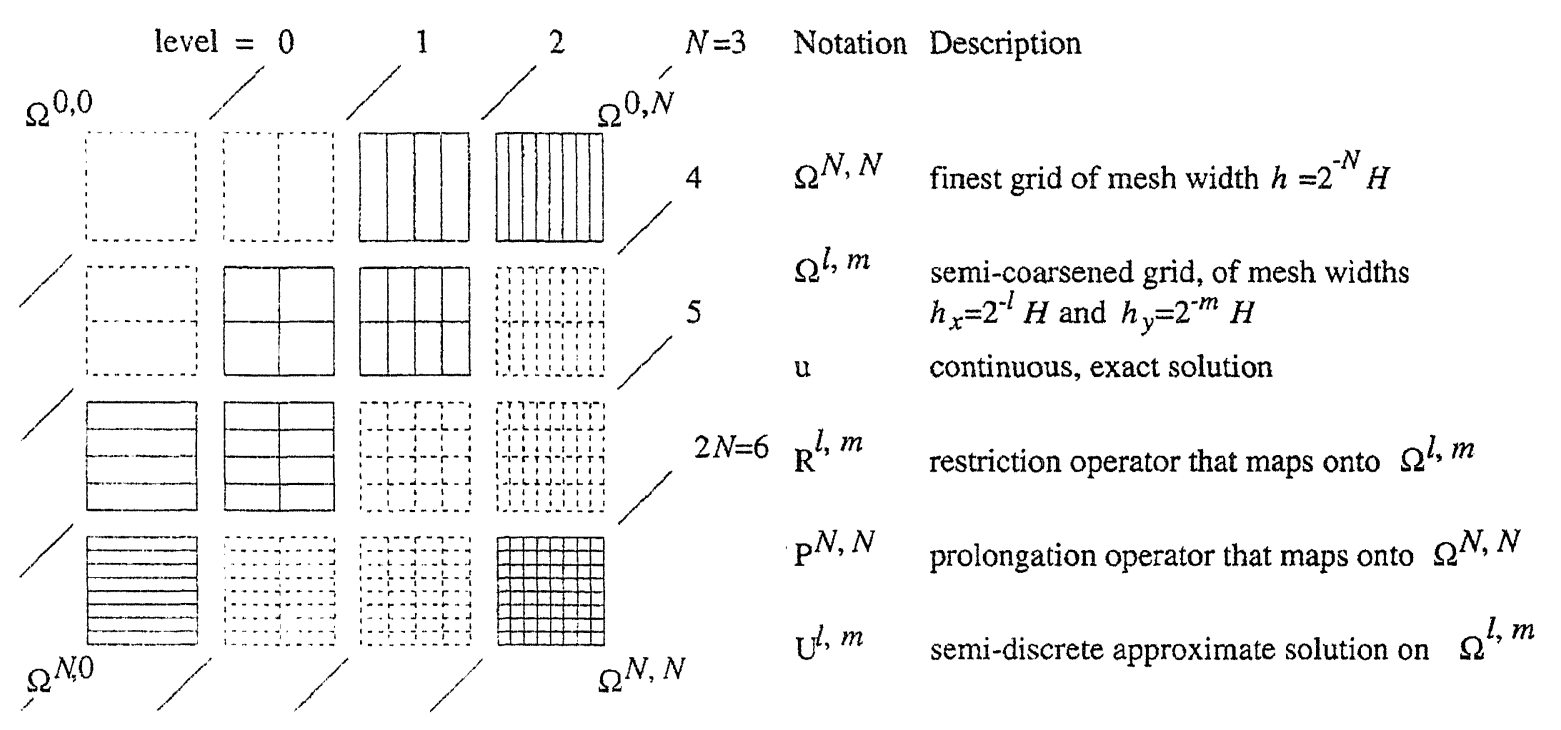

Figure 4. Grid of grids.

Analogously, assuming exact time integration and semi-discrete solutions $U^{l, m}$, resulting from a spatial discretization, the combination technique constructs an approximate solution $\widehat{U}^{N, N}$ on the finest grid $\Omega^{N, N}$ from the coarse-grid approximate solutions according to

$$
\widehat{U}^{N, N}=\sum_{l+m=N} P^{N, N} U^{l, m}-\sum_{l+m=N-1} P^{N, N} U^{l, m} .
$$

Let $d^{l, m}$ denote the discretization error on grid $\Omega^{l, m}$, i.e.,

$$
d^{l, m} \equiv U^{l, m}-R^{l, m} u,
$$

Then the total error $e^{N, N}=\widehat{U}^{N, N}-R^{N, N} u$ in $\widehat{U}^{N, N}$ is written as

$$
e^{N, N}=r^{N, N}+\widehat{d}^{N, N}
$$

where the combined discretization error $\widehat{d}^{N, N}=\widehat{U}^{N, N}-\widehat{u}^{N, N}$ is given by

$$
\widehat{d}^{N, N}=\sum_{l+m=N} P^{N, N} d^{l, m}-\sum_{l+m=N-1} P^{N, N} d^{l, m} .
$$

In [8] the representation error $r^{N, N}$ is analyzed, and in [9] an analysis is given of the combined discretization error $\widehat{d}^{N, N}$ for pure advection problems. In the next section we give similar results for the combined discretization error for our model problem 1 given by (2.1).

\section{Spatial discretization errors}

For the test problem (2.1), the linear constant-coefficient advection-diffusion problem, we can derive an expansion in $h_{x}$ and $h_{y}$ for the spatial discretization error, as we did for the pure advection problem in [9]. Since essentially the same approach is used as in [9] we state only the results. We consider the error in the semi-discrete solution due to spatial discretization only, i.e., we assume exact time integration. In (2.1a) the diffusion terms are discretized by second-order central differences and the advection term is discretized by the third-order upwind biased discretization [6]. We neglect the influence of boundary conditions, i.e., we 
only consider the error away from the boundaries. When solved on a single grid with mesh widths $h_{x}$ and $h_{y}$, the spatial discretization error can be formally expanded as

$$
\begin{aligned}
d(x, y, t) & =\sum_{i=1}^{\infty} \frac{\left(-t E_{\mathrm{adv}}-t E_{\mathrm{diff}}\right)^{i}}{i !} u(x, y, t), \\
E_{\mathrm{adv}} & =\sum_{j=3}^{\infty} \frac{-(-2)^{j}+3(-1)^{j}+1}{3(j+1) !} h_{x}^{j} \partial_{x}^{j+1}, \\
E_{\mathrm{diff}} & =\varepsilon \sum_{j=2}^{\infty} \frac{(-1)^{j}+1}{(j+2) !}\left(h_{x}^{j} \partial_{x}^{j+2}+h_{y}^{j} \partial_{y}^{j+2}\right),
\end{aligned}
$$

assuming that $u(x, y, t)$ is a $C^{\infty}$ function. Neglecting $O\left(h_{x}^{4}\right)$ and $O\left(h_{y}^{4}\right)$ but including $O\left(h_{x}^{2} h_{y}^{2}\right)$ for later comparison yields the following leading order expression

$$
\begin{aligned}
d(x, y, t)= & -\frac{t \varepsilon}{12}\left(h_{x}^{2} \partial_{x}^{4}+h_{y}^{2} \partial_{y}^{4}\right) u(x, y, t)-\frac{t}{12} h_{x}^{3} \partial_{x}^{4} u(x, y, t) \\
& +\frac{t^{2} \varepsilon^{2}}{144} h_{x}^{2} h_{y}^{2} \partial_{x}^{4} \partial_{y}^{4} u(x, y, t)+\mathcal{O}\left(h_{x}^{4}\right)+\mathcal{O}\left(h_{y}^{4}\right) .
\end{aligned}
$$

As in [9], we use this result to determine the resulting spatial discretization error in the combined solution. It is given by

$$
\begin{aligned}
\widehat{d}(t)= & -\frac{t \varepsilon h^{2}}{12}\left(\partial_{x}^{4}+\partial_{y}^{4}\right) u(t)-\frac{t h^{3}}{12} \partial_{x}^{4} u(t) \\
& +\frac{t^{2} \varepsilon^{2}}{144} H^{2} h^{2}\left(1-3 \log _{2} \frac{H}{h}\right) \partial_{x}^{4} \partial_{y}^{4} u(t)+\mathcal{O}\left(h^{3} \log _{2} \frac{1}{h}\right) .
\end{aligned}
$$

The first error term is the usual leading error term on $\Omega^{N, N}$ coming from the diffusion operator. Similarly, the second term stems from the advection operator. The third term originates from the mixing of diffusion in the $x$ - and $y$-direction in the CT-process. Since there is only advection in the $x$-direction, advection does not produce any additional error in the combined solution. In order for the CT to be effective, the third term should be small compared to the first two terms. Asymptotically (as $h$ and $H$ tend to zero) this is clearly the case. In practice, $h$ and $H$ are not always small enough for the third term (and higher order mixed terms) to be negligible.

\section{The Rosenbrock solver ROS3}

We consider autonomous ODE systems of the form

$$
\frac{d U}{d t}=f(U)
$$

which result from spatial discretization on one of our grids and we seek a numerical approximation $U_{n} \approx U(t)$ at $t=t_{n}$. To obtain this approximation we apply a third-order consistent two-stage Rosenbrock method, ROS3 (also used in [7]), which can be written as

$$
\begin{aligned}
U_{n+1} & =U_{n}+\frac{5}{4} k_{1}+\frac{3}{4} k_{2} \\
(I-\gamma \tau A) k_{1} & =\tau F\left(U_{n}\right) \\
(I-\gamma \tau A) k_{2} & =\tau F\left(U_{n}+\frac{2}{3} k_{1}\right)-\frac{4}{3} k_{1},
\end{aligned}
$$


where $\tau=t_{n+1}-t_{n}$ is the step size, and $A$ is the Jacobian matrix $f^{\prime}\left(U_{n}\right)$ or an $O(\tau)$ accurate approximation thereof. This scheme is a variation to the ROS2 scheme presented in [13] and belongs to a family of schemes discussed on p. 233 of [2]. Its stability function is

$$
R(z)=\frac{1+(1-2 \gamma) z+\left(\frac{1}{2}-2 \gamma+\gamma^{2}\right) z^{2}}{(1-\gamma z)^{2}}
$$

which shows that the scheme is A-stable if and only if $\gamma \geqslant 1 / 4$. The scheme is third-order accurate provided $\gamma=1 / 2+\sqrt{3} / 6$. Note that this specific $\gamma$ yields A-stability. Because our spatially discrete problems are stiff due to the diffusion term, A-stability is a desirable property,

\subsection{Factorization}

Since the ROS3 scheme remains of third-order for any $O(\tau)$ perturbation to $A=f^{\prime}\left(U_{n}\right)$, we can split $A$ as $A=A_{1}+A_{2}$ and use

$$
\begin{aligned}
U_{n+1} & =U_{n}+\frac{5}{4} k_{1}+\frac{3}{4} k_{2}, \\
\left(I-\gamma \tau A_{1}\right)\left(I-\gamma \tau A_{2}\right) k_{1} & =\tau F\left(U_{n}\right) \\
\left(I-\gamma \tau A_{1}\right)\left(I-\gamma \tau A_{2}\right) k_{2} & =\tau F\left(U_{n}+\frac{2}{3} k_{1}\right)-\frac{4}{3} k_{1} .
\end{aligned}
$$

The latter, factorized ROS3 scheme, is still of third-order since

$$
\left(I-\gamma \tau A_{1}\right)\left(I-\gamma \tau A_{2}\right)=I-\gamma \tau\left(A-\gamma \tau A_{1} A_{2}\right) .
$$

In the current work we use directional factorization, separating the horizontal and vertical coupling, such that $A_{1}$ only couples unknowns in the horizontal direction and $A_{2}$ only in the vertical direction. This leads to enormous savings in the required computational work since it reduces the two-dimensional linear algebra to one-dimensional linear algebra.

Without factorization, spatial discretization leads to $p q$ coupled linear algebraic equations for the Rosenbrock vectors $k_{1}$ and $k_{2}$ where $p$ is the number of unknowns in horizontal direction and $q$ the number in vertical direction. With factorization, we have only $p$ sets of $q$ coupled equations and $q$ sets of $p$ coupled equations for $k_{1}$ and for $k_{2}$. Moreover, the resulting sets of equations have band diagonal matrices and are therefore solved very efficiently by LU decomposition.

In [7] it has been proven that a similar property as A-stability holds for the factorized ROS3 scheme. For our model problems this means that we have unconditional stability in the sense of Fourier-Von Neumann.

\subsection{Time step size control}

In order to obtain an estimate for the local time error, in our implementation of ROS3 we compute an additional vector, $k_{3}$. The corresponding extra auxiliary equation is

$$
\left(I-\gamma \tau A_{1}\right)\left(I-\gamma \tau A_{2}\right) k_{3}=\tau F\left(U_{n+1}\right)+\frac{24 \gamma^{2}-9 \gamma-1}{6 \gamma(1-2 \gamma)} k_{1}+\frac{3 \gamma-1}{2 \gamma(1-2 \gamma)} k_{2} .
$$

The error estimate is

$$
\begin{aligned}
E_{\text {est }} & =-\frac{6 \gamma^{2}-1}{6 \gamma(1-2 \gamma)} k_{1}+\frac{6 \gamma^{2}-6 \gamma+1}{2 \gamma(1-2 \gamma)} k_{2}-k_{3} \\
& =\frac{1}{6} \tau^{3} \frac{d^{3} c}{c t^{3}}+O\left(\tau^{4}\right)
\end{aligned}
$$


which is the last term in the Taylor expansion of the updated solution that our scheme still handles correctly. Striving for an equidistribution of errors we attempt to keep $E_{\text {est }}$, measured in the $L_{1}$ norm, fixed at some tolerance Tol during the integration. To achieve this we adjust the step size $\tau$ according to

$$
\tau_{\text {new }}=0.8 \tau_{\text {old }}\left(\frac{\text { Tol }}{\left\|E_{\text {est }}\right\|_{1}}\right)^{1 / 3} .
$$

Solution updates are only performed when $\left\|E_{\text {est }}\right\|_{1} \leqslant$ Tol at the new time level, otherwise the update is rejected and recomputed with a smaller step size. The factor 0.8 is a safety factor and serves to avoid excessive numbers of rejected updates. In our implementation the ratio $\tau_{\text {new }} / \tau_{\text {old }}$ was kept bounded between 0.1 and 10 .

Now consider the global time error $e_{n}$ at the time level $t_{n}$, i.e., the difference between the computed solution at the time level $t_{n}$ and the exact solution at the same time level. This error is proportional to the tolerance Tol that we imposed, i.e.,

$$
e_{n} \sim \text { Tol. }
$$

This property of tolerance proportionality follows from [12], p. 350, when we identify our scheme as an XEPS scheme, i.e., an error per step control with local extrapolation. The proportionality between the imposed tolerance and the global time error is a nice property since it allows the user to control the global error in a very direct manner.

\subsection{Numerical illustration of factorized ROS3}

Figure 5 displays the integration history for the Burgers' equations solved up to $t=3$ on a single $33 \times 33$ spatial grid with $\mathrm{Tol}=10^{-3}$. The step size $\tau$ is shown in the left graph and the error estimate $\left\|E_{\text {est }}\right\|_{1}$ in the right graph. We start with an initial step size $\tau=10^{-2}$ which turns out to be somewhat too small for the imposed tolerance value. As the integration proceeds, larger step sizes are permitted. In the intermediate stage of the integration, the step size remains almost constant. Finally, as the solution approaches the steady state, the size of the allowed step size quickly grows. During the integration the step size control keeps the error estimate $\left\|E_{\text {est }}\right\|_{1}$ at a nearly constant level, as can be seen from Fig. 5.

In Table 1 the ratio is shown of maximal global time errors $E_{\mathrm{Tol}}$ for $\mathrm{SG}$ solutions with tolerance Tol and tolerance Tol/2 as a function of the tolerance Tol. The time errors were estimated by subtracting a reference solution obtained with $\mathrm{Tol}=10^{-8}$. As the tolerance, and hence the step size, gets smaller, we see that the ratio approaches 2 , which confirms that the global time error is proportional to the imposed tolerance. The errors $E_{\text {Tol }}$ are measured in the discrete $L_{1}$ norm on a $33 \times 33$ grid at $t=3$.

Table 1. Ratio of global time errors for model problem 2

\begin{tabular}{|c|c|}
\hline Tol & $L_{\infty}\left(E_{\mathrm{Tol}}\right) / L_{\infty}\left(E_{\mathrm{Tol} / 2}\right)$ \\
\hline $10^{-3}$ & 1.748 \\
\hline $10^{-4}$ & 1.597 \\
\hline $10^{-5}$ & 1.878 \\
\hline $10^{-6}$ & 1.973 \\
\hline
\end{tabular}



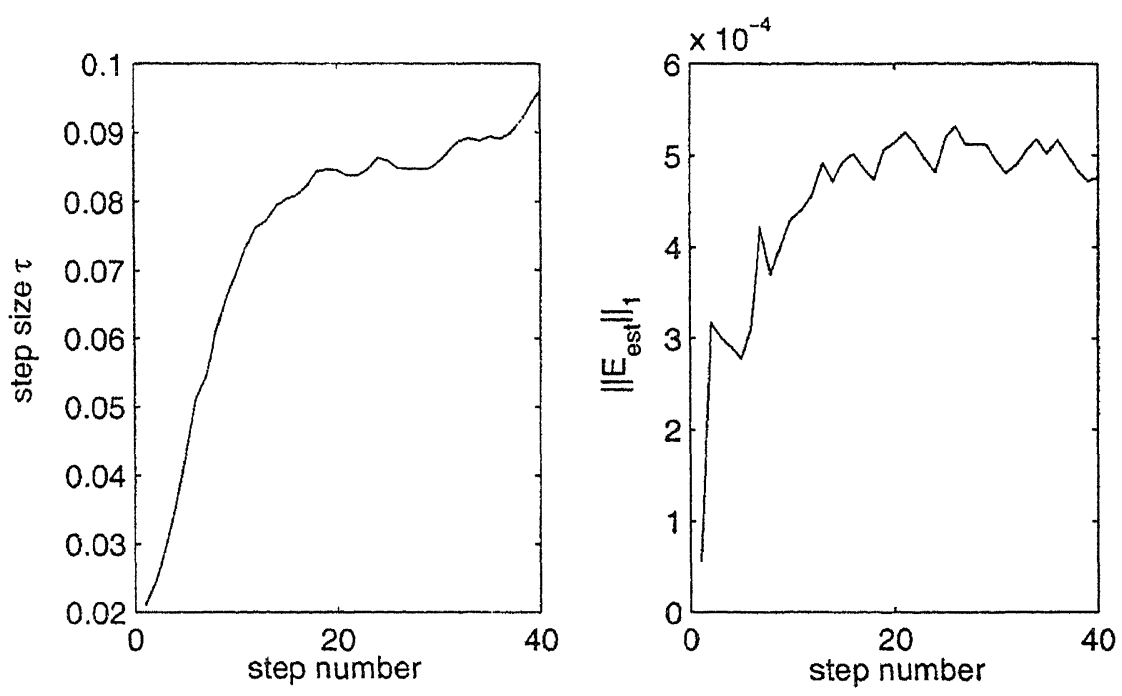

Figure 5. Integration history of model problem 2

\section{Results}

In this section the CT is compared with the standard SG approach. Both are implemented with the same spatial discretization, i.e., second-order central discretization for the diffusion operator and third-order upwind-biased discretization for the advection part. The Neumann condition at the outflow boundary in model problem $1(2.1)$ is only imposed on the diffusion operator to avoid spurious reflections.

\subsection{Validation of the sparse grid error expression}

In Fig. 6 a numerical illustration of the sparse grid error behaviour is given. Spatial errors are shown for solutions of (2.1a) with initial profile

$$
u(x, y, 0)=e^{-16\left(x^{2}+y^{2}\right)}
$$

integrated up to $t=0.25$, with $\varepsilon=0.05$ and zero Dirichlet boundary conditions. A sparse grid with $N=5$, i.e., containing 11 semi-coarsened grids, was used. The top row of Fig. 6 corresponds to the solutions obtained with a root mesh width $H=1 / 2$, the bottom row corresponds to $H=1 / 8$. The errors in the left column were obtained numerically, i.e., by subtracting the reference solution obtained on a finer grid $(N=5, H=1 / 32)$. The errors in the right column are predictions according to (4.1) where the derivatives of the solution were replaced by numerical differences of the reference solution.

The errors in the top row show oscillatory behaviour that is due to the third term in (4.1), i.e., the term due to combination. This behaviour is absent in the lower row. Here the third term, which is proportional to $H^{2}$, can be neglected due to the smaller $H=1 / 8$. The error prediction (4.1) illustrated in the right column clearly matches this transition in error behaviour.

\subsection{Model problem 1: the advection diffusion equation}

In Fig. 7 the efficiency of the CT is compared with the SG when applied to the linear constant-coefficient advection-diffusion equation. Along the vertical axes the discrete $L_{1}$ error meausured on $\Omega^{N, N}$ is plotted in the left column of graphs and the discrete $L_{\infty}$ error 


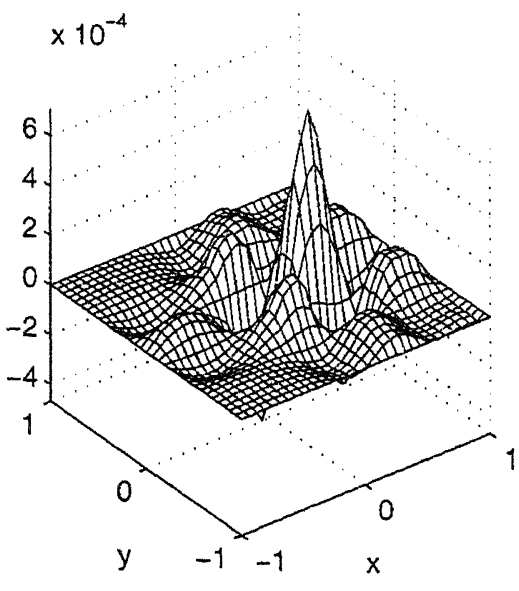

Observed error, $\mathrm{H}=1 / 2$

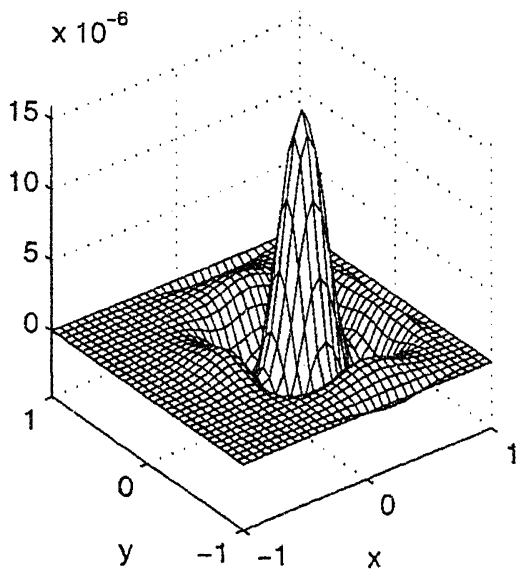

Observed error, $\mathrm{H}=1 / 8$

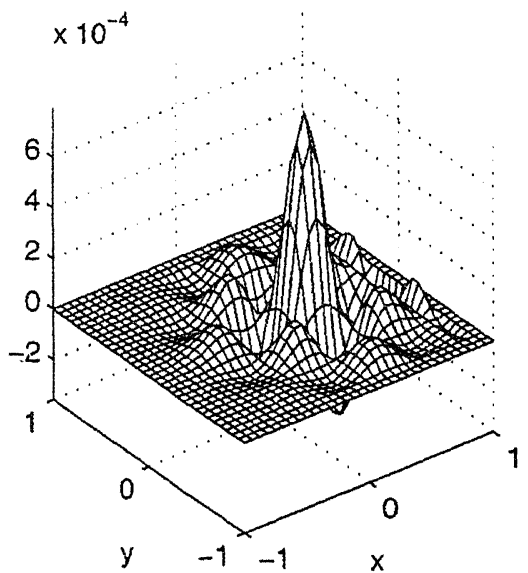

Predicted error, $\mathrm{H}=1 / 2$

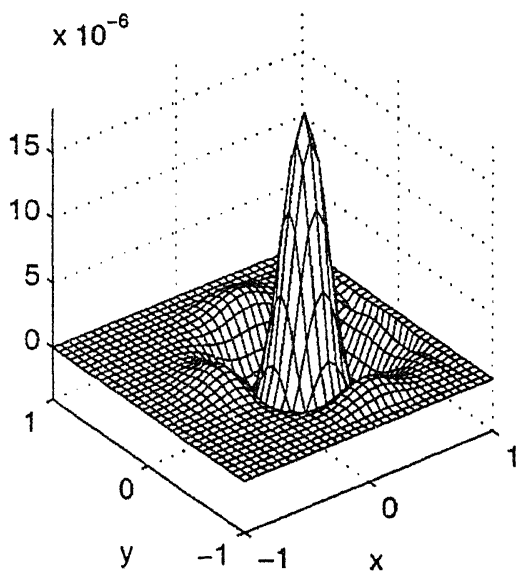

Predicted error, $\mathrm{H}=1 / 8$

Figure 6. Spatial errors

is plotted in the right column. Along the horizontal axes the computational work is plotted in terms of the number of required cell updates. The graphs in the top, middle and bottom row correspond to $\varepsilon=10^{-2}, 10^{-3}$ and $10^{-5}$, respectively.

We see that for all these $\varepsilon$ the CT is more efficient than the SG if we consider the errors in the $L_{1}$ norm. Also, the gain in efficiency becomes larger as $\varepsilon$ decreases. This is expected since for small $\varepsilon$ the grid-aligned advection becomes more dominant rendering the test case more grid-aligned and hence better suited to the CT. For $\varepsilon=10^{-3}$ and $10^{-5}$ the same holds for the $L_{\infty}$ norm. For $\varepsilon=10^{-2}$ the CT does not perform well when measured in the $L_{\infty}$ norm. Examination of the corresponding spatial error distribution shows that the maximum error occurs near the discontinuity in the inlet condition. The mixed derivative $u_{x x y y}$ is large near this discontinuity which causes, for large $\varepsilon$, a large term $\varepsilon^{2} u_{x x y y}$ in the spatial error for the CT. Hence, it is to be expected that for relatively large $\varepsilon$ the CT performs poorly locally near the discontinuity. 

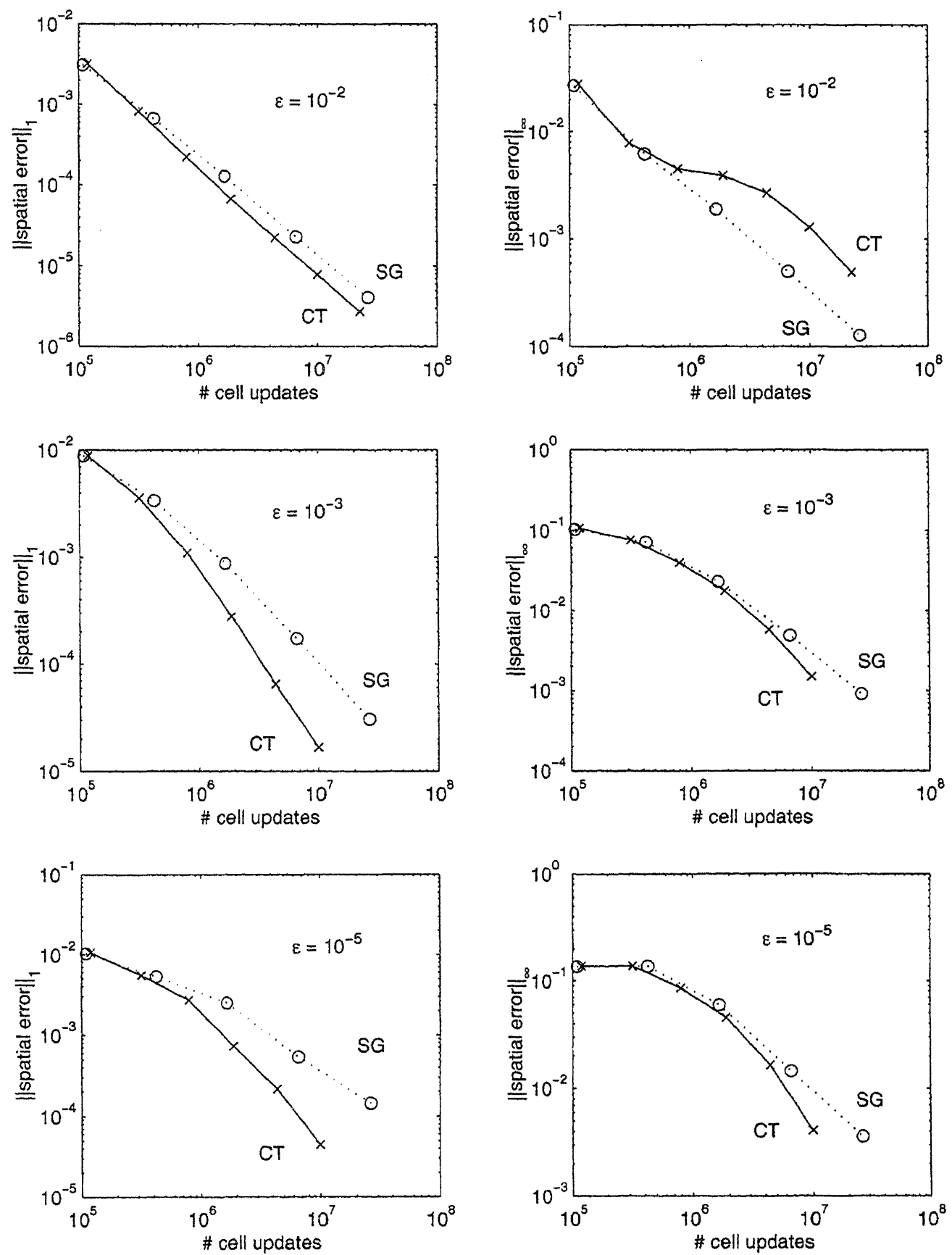

Figure 7. Efficiency comparisons for model problem 1.

\subsection{Model problem 2: Burgers' equations}

In Fig. 8 the CT and SG are compared for the 2D Burgers' test case. In Fig. 8 the diffusion parameter is kept fixed at $\varepsilon=10^{-2}$ because it appears that varying the diffusion parameter (between $10^{-5}$ and $10^{-1}$ ) does not change the qualitative conclusions that can be drawn. The top row corresponds to the Burgers' test problem described in Section 2.2. For this test case it is clear that CT does not perform well compared with SG, when measured either in $L_{1}$ norm or in $L_{\infty}$ norm. It was expected that the Burgers' test case would be less well suited 
to the CT than the linear test case since the solution of the former is less grid-aligned.

To see how the CT performs on the Burgers' test case when it is more grid-aligned, we take as initial condition $v=0$, which guarantees that $v$ remains zero. Furthermore we replace the parabolic inlet condition by

$$
u(-1, y, t)=\left\{\begin{array}{l}
\cos ^{2}(y-1 / 2), y \geqslant 0, \\
\cos ^{2}(y+1 / 2)^{2}, y<0 .
\end{array}\right.
$$

This removes a strong peak in the error at $(x, y)=(-1,0)$ which would otherwise dominate the error. The results for this reduced Burgers' test case are shown in the bottom row of Fig. 8. Measured in the $L_{1}$ norm the CT outperforms a SG when applied to this reduced test case. However, measured in the $L_{\infty}$ norm this is still not the case, but both solutions are comparable.
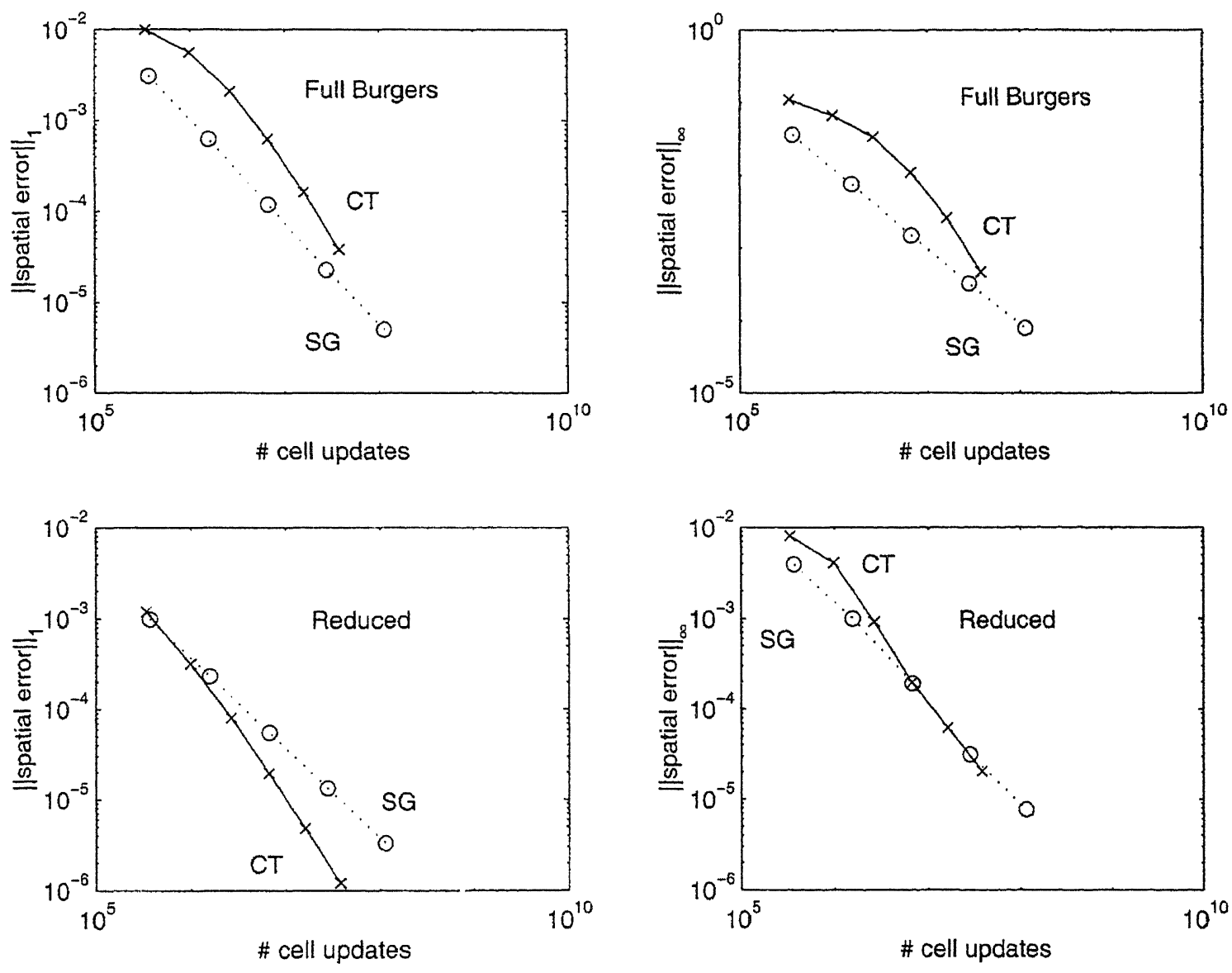

Figure 8. Efficiency comparisons for model problem 2

\section{Conclusions}

When applied to the simple grid-aligned, linear constant-coefficient test case the CT is clearly superior to the SG approach in terms of efficiency. Especially when the diffusion parameter $\varepsilon$ is small, the linear test case is strongly grid-aligned and very well suited for the CT.

When applied to the 2D Burgers' test case, the CT does not perform so well. However, the CT does perform reasonably well for a reduced version of the Burgers' test case when advection appears in only one direction. 
Based on these observations, our expectation that the CT is well suited to advectiondiffusion problems that are strongly grid-aligned has been confirmed. But it seems that the $\mathrm{CT}$ is less suited to more general problems where the solution features are not well aligned with the grid.

\section{References}

[1] H. J. Bungartz, M. Griebel, D. Roschke, and C. Zenger, Pointwise convergence of the combination technique for the laplace equation, East-West J. Numer. Math., 2 (1994), No. 1, pp. 21-45.

[2] K. Dekker and J. G. Verwer, Stability of Runga-Kutta Methods for Stiff Nonlinear Differential Equations, Elsevier North-Holland, Amsterdam, 1984.

[3] M. Griebel, The combination technique for the sparse grid solution of pde's on multiprocessor machines, Parallel Processing Letters, 2 (1992), No. 1, pp. 61-70.

[4] M. Griebel, M. Schneider, and C. Zenger, A combination technique for the solution of sparse grid problems, in: Iterative Methods in Linear Algebra (R. Beauwens and P. de Groen, eds.), North-Holland, Amsterdam, 1992, pp. 263-281.

[5] M. Griebel and G. Zumbusch, Adaptive sparse grids for hyperbolic conservation laws, in: Notes on Numerical Fluid Mechanics (W. Hackbusch and G. Wittum, eds.), Vieweg, Braunschweig, 1999, .

[6] B. Koren, A robust upwind discretization method for advection, diffusion and source terms, in: Numerical Methods for Advection-Diffusion Problems (C. B. Vreugdenhil and B. Koren, eds.), vol. 45 of Notes on Numerical Fluid Mechanics, Vieweg, Braunschweig, 1993, pp. 117-138.

[7] D. Lanser, J. G. Blom, and J. G. Verwer, Time integration of the shallow water equations in spherical geometry, Report mas-r0021, CWI, Amsterdam, 2000, http://www.cwi.nl/static/publications/reports/MAS-2000.html.

[8] B. Lastdrager and B. Koren, Error analysis for function representation by the sparse-grid combination technique, Report mas-r9823, CWI, Amsterdam, 1998, http://www.cwi.nl/static/publications/reports/MAS-1999.html.

[9] B. Lastdrager, B. Koren, and J. G. Verwer, The sparse-grid combination technique applied to timedependent advection problems, Report mas-r9930, CWI, Amsterdam, 1999, http://www.cwi.nl/static/publications/reports/MAS-1999.html.

[10] B. Lastdrager, B. Koren, and J. G. Verwer, The sparse-grid combination technique applied to timedependent advection problems, in: Proceedings of the Sixth European Multigrid Conference, Gent, 1999 (E. Dick, K. Riemslagh, and J. Vierendeels, eds.), vol. 14 of Lecture Notes in Computational Science and Engineering, Springer, Berlin, 2000, pp. 143-149.

[11] U. Rüde, Multilevel, extrapolation and sparse grid methods, in: Multigrid Methods (P. W. Hemker and P. Wesseling, eds.), vol. IV, Birkhäuser, Basel, 1993, pp. 281-294.

[12] L. F. Shampine, Numerical Solution of Ordinary Differential Equations, Chapman \& Hall, New York, 1994.

[13] J. G. Verwer, E. J. Spee, J. G. Blom, and W. Hundsdorfer, A second-order rosenbrock method applied to photochemical dispersion problems, SIAM J. Sci. Comput., 20 (1999), No. 4, pp. 1456-1480.

[14] C. Zenger, Sparse grids, vol. 31 of Notes on Numerical Fluid Mechanics, Vieweg, Braunschweig, 1991, pp. 241-251. 